LBL-36398

\title{
Growth of Hydrogenated Amorphous Silicon (a-Si:H) on Patterned Substrates for Increased Mechanical Stability
}

\author{
Wan-Shick Hong, Juan Carlos Delgado Nieto, Oscar Ruiz \\ and Victor Perez-Mendez
}

Presented at the MRS Symp. Boston, MA, Nov. 28 - Dec. 2, 1994 and to be published in the Proceedings

\author{
Physics Division \\ Lawrence Berkeley Laboratory \\ University of California \\ Berkeley, CA 94720
}

December 1994

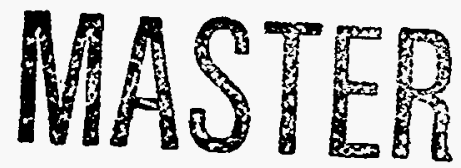

This work was supported by the Director, Office of Energy Research, Office of High Energy and Nuclear Physics, Division of High Energy Physics of the U. S. Department of Energy under Contract No. DE-AC03-76SF00098. 


\section{DISCLAIMER}

This report was prepared as an account of work sponsored by an agency of the United States Government. Neither the United States Government nor any agency thereof, nor any of their employees, make any warranty, express or implied, or assumes any legal liability or responsibility for the accuracy, completeness, or usefulness of any information, apparatus, product, or process disclosed, or represents that its use would not infringe privately owned rights. Reference herein to any specific commercial product, process, or service by trade name, trademark, manufacturer, or otherwise does not necessarily constitute or imply its endorsement, recommendation, or favoring by the United States Government or any agency thereof. The views and opinions of authors expressed herein do not necessarily state or reflect those of the United States Government or any agency thereof. 


\section{DISCLAIMER}

Portions of this document may be illegible in electronic image products. Images are produced from the best available original document. 


\title{
GROWTH OF HYDROGENATED AMORPHOUS SILICON (A-Si:H) ON PATTERNED SUBSTRATES FOR INCREASED MECHANICAL STABILITY
}

\author{
W.-S. HONG, J.C. DELGADO*, O. RUIZ* and V. PEREZ-MENDEZ \\ Physics Division, Lawrence Berkeley Laboratory, Berkeley, CA 94720, U.S.A. \\ *Dept. de Fisica Aplicada i Electronica, Universitat de Barcelona, Barcelona, Spain
}

\begin{abstract}
Residual stress in hydrogenated amorphous silicon (a-Si:H) film, which causes substrate bending and delamination, is studied. The internal stress can be reduced by controlling deposition parameters, but it is known to produce a trade-off between stress and electronic quality. Selective area deposition, in which the deposition area is reduced by making islands, reduced the stress when the lateral dimension of the islands becomes comparable to the film thickness. The overall stress is reduced by approximately $40 \%$ when the lateral dimension is decreased to $40 \mu \mathrm{m}$, but the adhesion was not improved much. However, substrates having a 2-dimensional array of inversed pyramids of $200 \mu \mathrm{m}$ in lateral dimension produced overall stress $3 \sim 4$ times lower than that on the normal substrates. Such substrates were prepared by anisotropic etching of silicon wafers. The inversed pyramid structure also has other advantages including minimized delamination and increased effective thickness. Computer simulation confirmed that the overall stress can be reduced by deposition on the pyramidal structure.
\end{abstract}

\section{INTRODUCTION}

Hydrogenated amorphous silicon (a-Si:H) is gaining increasing use in photovoltaic solar cells, facsimile heads, liquid crystal display drivers and radiation detectors. The special attribute of a$\mathrm{Si}: \mathrm{H}$ is the ability to deposit the material inexpensively over large areas and the high resistance to radiation damage. Radiation detection with a-Si:H layers has been investigated for applications in high energy physics experiments, medical imaging, material and life science.1), 2),3)

In order to detect minimum ionizing particles, $\mathrm{p}-\mathrm{i}-\mathrm{n}$ diodes with thick $(\sim 50 \mu \mathrm{m})$ i layers are needed in which the particle can produce a sufficient number of electron-hole pairs by direct interaction in the depleted i layer. ${ }^{4}$ ) Device quality a-Si:H films are made mostly by plasmaenhanced chemical vapor deposition (PECVD) technique. However, it takes long time to grow thick layers with a good electronic quality by the current technology and the layers have a large built-in stress. This stress causes the substrate bending which in turn degrades position accuracy of the detector, and sometimes the film peels off from the substrate. The objective of this research is to reduce these negative effects of the high compressive stress without affecting good electrical properties.

\section{BACKGROUND}

\section{Origin of the Stress}

The total residual stress in a-Si:H films is the sum of the internal stress and the thermal stress.5) The stress arising from thermal expansion mismatch is usually less than $10 \%$ of the total stress 


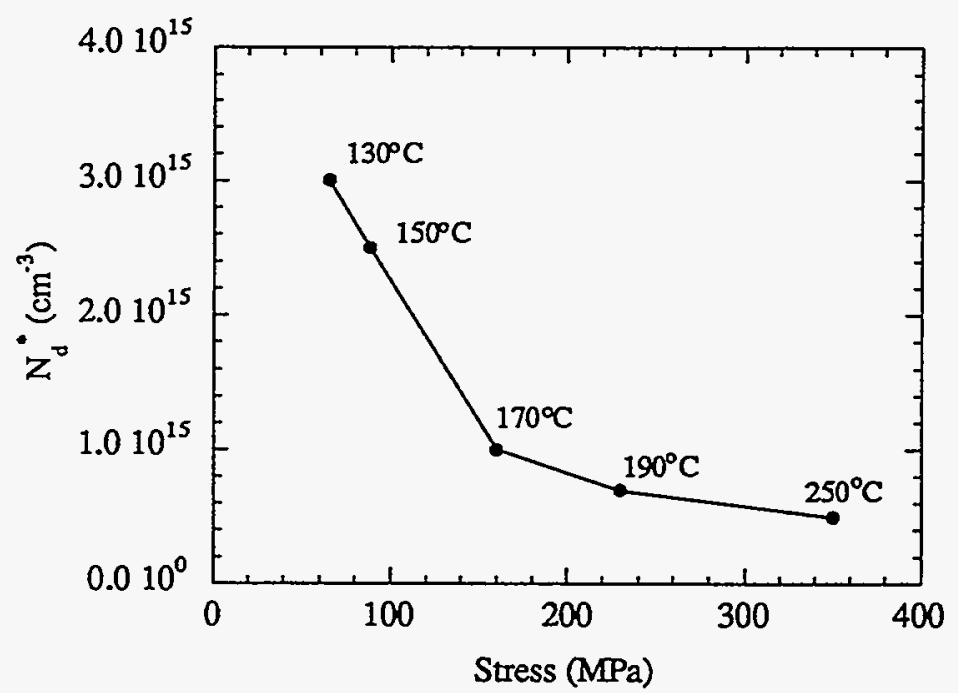

Fig. 1. Relationship between the residual stress and ionized dangling bond density $\left(\mathrm{N}_{\mathrm{d}}{ }^{*}\right)$ for a-Si:H films deposited at various temperatures. The deposition temperatures are indicated on each data points. with a temperature difference of $225^{\circ} \mathrm{C}$. Therefore, the observed residual stress is mainly due to the large internal compressive stress.

The origin of the internal compressive stress is not well understood. However, the hydrogen incorporation model proposed by Stevens and Johnson ${ }^{6)}$ and by Harbison et al.,7) is believed to be the major cause of the compressive stress. The first process for hydrogen incorporation occurs simultaneously with the addition of silicon atoms to the random network at the growing surface. During this process some hydrogen is incorporated as the network is forming, but the hydrogen incorporated in this process needs not contribute to intrinsic stress since its incorporation occurs integrally with the formation of the rigid silicon network at the growing surface.

However, the second process involves the invasive incorporation of hydrogen into the rigid random network and directly produces compressive intrinsic stress. Some excess hydrogen at the growing surface diffuses into the near surface region and chemically reacts with the rigid network. The hydrogen atom produces repulsive force between itself and its surrounding. The occurrence of this process and its consequences have been thoroughly established from studies of hydrogen migration in single-crystal silicon. ${ }^{8), 9)}$

\section{Defect Passivation by Hydrogen in a-Si:H}

The dangling bonds in the amorphous silicon network leads to a high density of deep traps in the band gap and thus degrades the electronic property of this material to a significant extent.10) When the atomic hydrogen is introduced, it terminates dangling bonds and therefore reduce the defect states from $\sim 10^{19}$ to $\sim 10^{15} \mathrm{~cm}^{-3}$ in the gap between the conduction and valence bands. Atomic hydrogen concentration of about $10 \%$ is known to be the optimum condition to make a device quality material.

The dangling bonds are ionized by releasing the non-bonded electrons under the electric field and become positively charged. This fixed positive charge causes the electric field in the i-layer to drop linearly with distance. Hence, in order to have a fully depleted detector, a minimum bias is needed which increases proportionately to the density of dangling bonds, and the ionized dangling bond density, $\mathrm{N}_{\mathrm{d}}{ }^{*}$, is the major quantity of interest to fabricate good detectors. The maximum ionized dangling bond density under strong reverse bias has been empirically determined as roughly one third of the total dangling bond density. Device quality a-Si:H has $\mathrm{N}_{\mathrm{d}}{ }^{*}$ of $5 \sim 10 \mathrm{x}$ $10^{14} \mathrm{~cm}^{-3}$.

\section{TECHNICAL APPROACH}




\section{Relationship between the stress and dangling bond density}

We prepared a series of p-i-n diodes with $5 \mu \mathrm{m}$-thick i-layer sandwiched in $0.1 \mu \mathrm{m}$-thick pand n-layers and measured the stress and $\mathrm{N}_{\mathrm{d}}{ }^{*}$ to see the relationship between them. The substrate temperature was varied between $130^{\circ} \mathrm{C}$ and $250^{\circ} \mathrm{C}$. All other conditions were fixed at the $\mathrm{RF}$ frequency of $85 \cdot \mathrm{MHz}, \mathrm{RF}$ power density of $40 \mathrm{~mW} / \mathrm{cm}^{2}$, chamber pressure of $300 \mathrm{mtorr}$. The total stress induced in a-Si:H films deposited on flat surfaces was calculated from eqn.1 by measuring the curvature of the substrate.11)

$$
\sigma_{\text {tot }}=\frac{E_{s}}{6\left(1-v_{s}\right)} \frac{t_{s}^{2}}{t_{f}} \frac{1}{R}
$$

where $E_{s}$ and $v_{s}$ are Young's modulus and Poisson's ratio of the substrates, respectively, $t_{s}$ and $t_{f}$ are the thicknesses of the substrate and a-Si:H film, and $\mathrm{R}$ is the radius of curvature of the substrate. The ionized dangling bond density was determined by hole onset measurement, in which the hole signal amplitude is measured while illuminating the $n$ - side of the $p-i-n$ diode at various applied bias voltages. The hole signal threshold bias corresponds to full depletion and is used to calculate the $\mathrm{N}_{\mathrm{d}}{ }^{*} \cdot{ }^{12)}$

The relationship between stress and $N_{d}{ }^{*}$ is plotted in Fig. 1. The deposition temperatures are indicated for each data points. The stress increased monotonously with decreasing $\mathrm{N}_{\mathrm{d}}{ }^{*}$. This result agrees well with other work which indicated that low stress materials have high $\mathrm{N}_{\mathrm{d}}{ }^{*}$ or vice versa.13) The kink at the substrate temperature of $170^{\circ} \mathrm{C}$ indicates that both the stress and $\mathrm{N}_{\mathrm{d}}{ }^{*}$ undergo an abrupt transition around this temperature. It is consistent with other results that silicon dihydride bond $(=\mathrm{Si}<\mathrm{H})$ formation sets in below $190^{\circ} \mathrm{C}$, which indicates the existence of

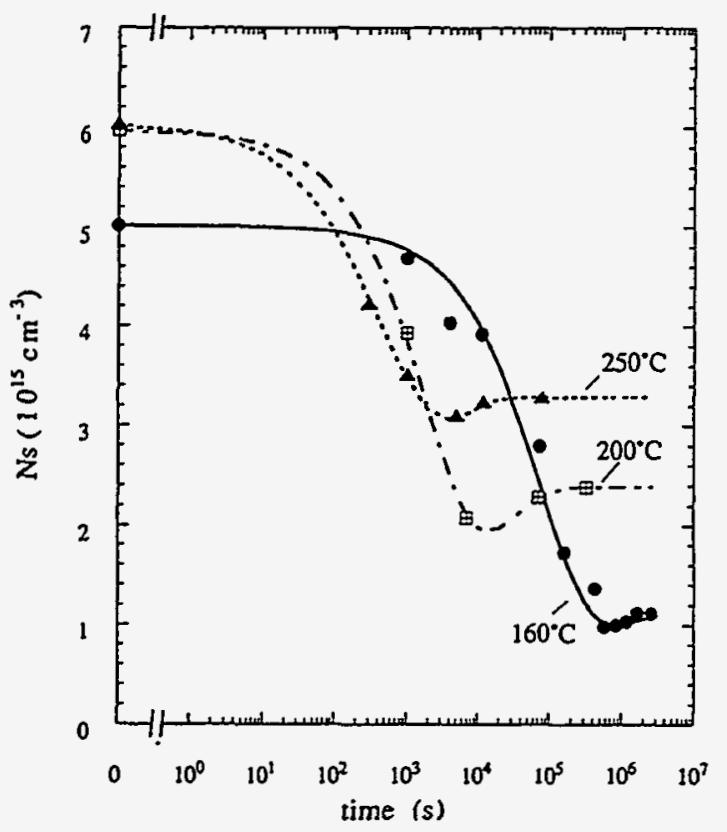

(a)

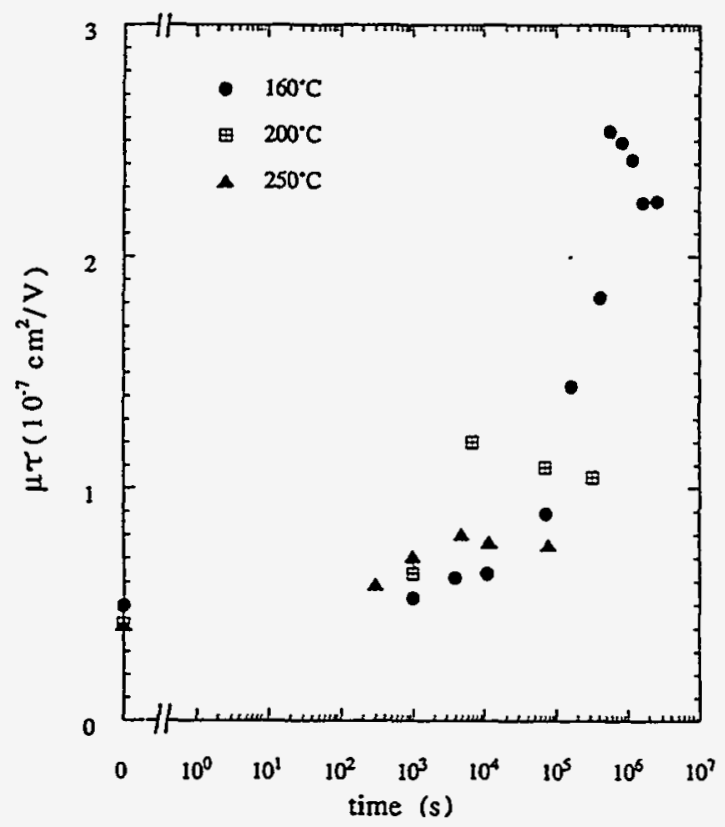

(b)

Fig. 2. Time dependence or (a) spin density and (b) normalized residual stress during annealing at different temperatures. The samples were degraded by heating at $300^{\circ} \mathrm{C}$ for 10 minutes and quenched in water prior to annealing. The stress values are normalized to the stress of as-deposited sample. 


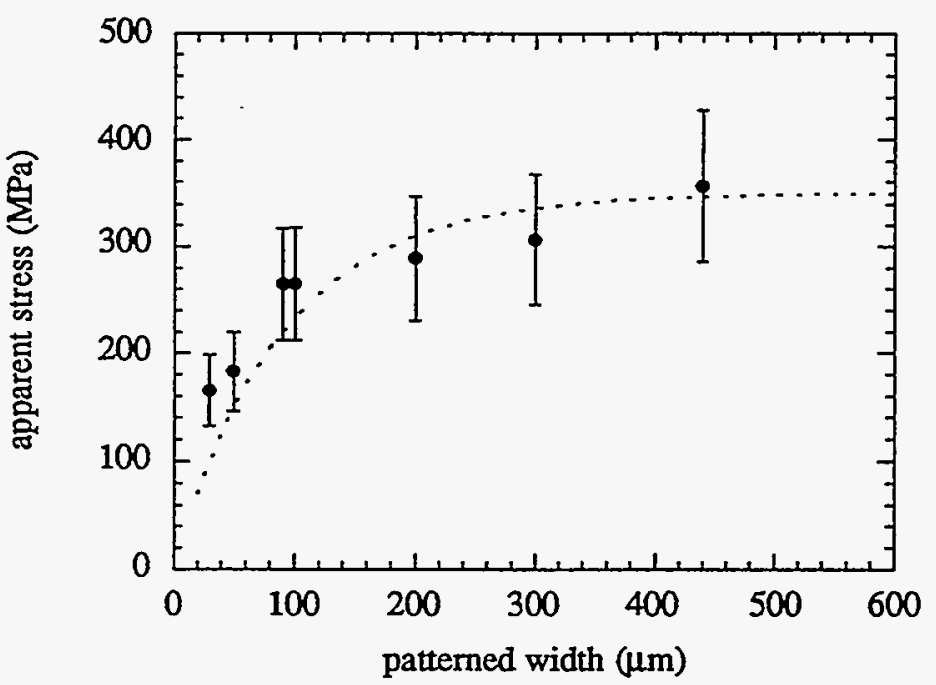

Fig. 3. Comparison of calculated and experimental results for stress reduction in the a-Si:H films grown on square islands as a function of the lateral dimension of the squares. The dashed line and closed circles represent calculated and experimental data, respectively. microvoids. ${ }^{14)}$

For standard a-Si:H films, the increased $\mathrm{N}_{\mathrm{d}}{ }^{*}$ can be mostly recovered by annealing at a proper temperature without affecting the stress appreciably.15) In fig. 2, changes in spin density and normalized stress with annealing time are plotted for a-Si:H samples which were prepared at $250^{\circ} \mathrm{C}$, degraded by heating at $300^{\circ} \mathrm{C}$, and annealed at various temperatures. It is seen that the spin density decreased from $5 \times 10^{15} \mathrm{~cm}^{-3}$ to $1 \times$ $10^{15} \mathrm{~cm}^{-3}$ after $\sim 100$ hours of annealing at $160^{\circ} \mathrm{C}$ and that the stress remained constant with annealing within the experimental error.

Selective Area Deposition - Square Islands

In order to avoid degrading the electronic quality of the a-Si:H by changing the deposition conditions,

we attempted to find some ways to minimize the negative effects of the stress, such as substrate bending and delamination, instead of reducing the film stress itself. One possible approach is to control the substrate morphology. By patterning the substrate, space for strain relaxation can be provided, or the net stress imposed on the substrate can be lowered.16), 17) Furthermore, by dividing the film into 'pixels', we can make a 2-dimensional array for a position sensitive detector.

The first attempt was to pattern the substrate into small square islands by making grooves chemically or mechanically. This selective area growth is reported to reduce the thermally induced residual stress in GaAs/Si heteroepitaxy system according to the following equation ${ }^{16), 18 \text { ) }}$

$$
\sigma=\sigma_{0}[1-\exp \{-k(w-x)\}]
$$

where $\sigma$ and $\sigma_{o}$ are residual stress for selective area growth and normal growth, respectively, $w$ is half the patterned width, $\mathrm{x}$ is the distance from the center of the patterned film, and $\mathrm{k}$ is the interfacial compliance parameter given by:

$$
\begin{aligned}
& \mathrm{k}^{2}=\frac{1}{\mathrm{k}_{\mathrm{o}}}\left\{\frac{1-v_{\mathrm{f}}^{2}}{\mathrm{E}_{\mathrm{f}} \mathrm{t}_{\mathrm{f}}}+\frac{1-v_{\mathrm{s}}^{2}}{\mathrm{E}_{\mathrm{s}} \mathrm{t}_{\mathrm{s}}}+\frac{3\left(1-v_{\mathrm{f}}^{2}\right)\left(1-v_{\mathrm{s}}^{2}\right)\left(\mathrm{t}_{\mathrm{f}}+\mathrm{t}_{\mathrm{s}}\right)^{2}}{\mathrm{E}_{\mathrm{f}} \mathrm{t}_{\mathrm{f}}^{3}\left(1-v_{\mathrm{s}}^{2}\right)+\mathrm{E}_{\mathrm{s}} \mathrm{t}_{\mathrm{s}}^{3}\left(1-v_{\mathrm{f}}^{2}\right)}\right\} \\
& \text { and } \mathrm{k}_{\mathrm{o}}=\frac{2}{3}\left\{\frac{\left(1+v_{\mathrm{f}}\right) \mathrm{t}_{\mathrm{f}}}{\mathrm{E}_{\mathrm{f}}}+\frac{\left(1+v_{\mathrm{s}}\right) \mathrm{t}_{\mathrm{s}}}{\mathrm{E}_{\mathrm{s}}}\right\}
\end{aligned}
$$

The above equation implies that the stress in the film becomes maximum at the center $(x=0)$ and drops exponentially near the edge $(\mathrm{x}=\mathrm{w})$ of the patterned mesas. Therefore, if the patterned width is small enough to offset the large $k$, the maximum stress in the film will be decreased by exp(kw). 
However, since the thickness and the stress distribution for patterned substrates are not uniform across the substrate, eqn. (1) cannot be used to estimate the stress in the patterned substrate. Also, since the films is amorphous, other stress measurement techniques, such as photoluminescence or X-ray diffraction, cannot be applied. Therefore, we used an equivalent thickness, $t_{s, \text { eqv }}$, which is a thickness of an imaginary, plain substrate having the same volume and area of the patterned substrate, in place of the substrate thickness in eqn. (1). The resulting "apparent stress", is the stress corresponding to a curvature of a plain substrate of thickness $t_{s,}$ eqv. and can be represented as follows:

$$
\sigma_{\text {app }}=\frac{E_{s}}{6\left(1-v_{s}\right)} \frac{t_{s, \text { eqv }}^{2}}{t_{f}} \frac{1}{R}
$$

The single crystalline $\mathrm{Si}$ wafer coated with a thin $(\sim 500 \AA)$ layer of $\mathrm{Cr}$ was chosen as a substrate because of good adhesion, surface uniformity, and convenience for further processing. The thin $\mathrm{Cr}$ layer is known to improve adhesion of a-Si:H film to the substrate without changing overall stress state significantly, and forms a quasi-ohmic contact with both $\mathrm{n}$ - and p-type layers. ${ }^{10)}$ Trenches of $80 \mu \mathrm{m}$ in width and $100 \mu \mathrm{m}$ in depth were cut on the surface of Si wafers using a diamond saw to make square mesas of lateral dimensions ranging from $40 \mu \mathrm{m}$ to $440 \mu \mathrm{m}$. The patterned substrate was then sputter coated with $\mathrm{Cr}$, and a-Si:H films of up to $32 \mu \mathrm{m}$ in thickness were deposited. The deposition parameters were chosen from fig. 1 such that a dangling bond density as low as possible $\left(\mathrm{N}_{\mathrm{d}}{ }^{*}=5 \times 10^{14} \mathrm{~cm}^{-3}\right)$ was obtained. For comparison, films were also grown on flat surfaces. The substrate curvature was measured by scanning the backside of the wafer with Alphastep 200 Profilometer. The fractured cross section was observed by scanning electron microscopy (SEM).

Fig. 3 shows the stress values estimated from the substrate curvature and theoretical values calculated from eqn. (2). The apparent stress decreased exponentially as the lateral dimension of the mesas decreased. Approximately $40 \%$ stress reduction was achieved when the patterned width

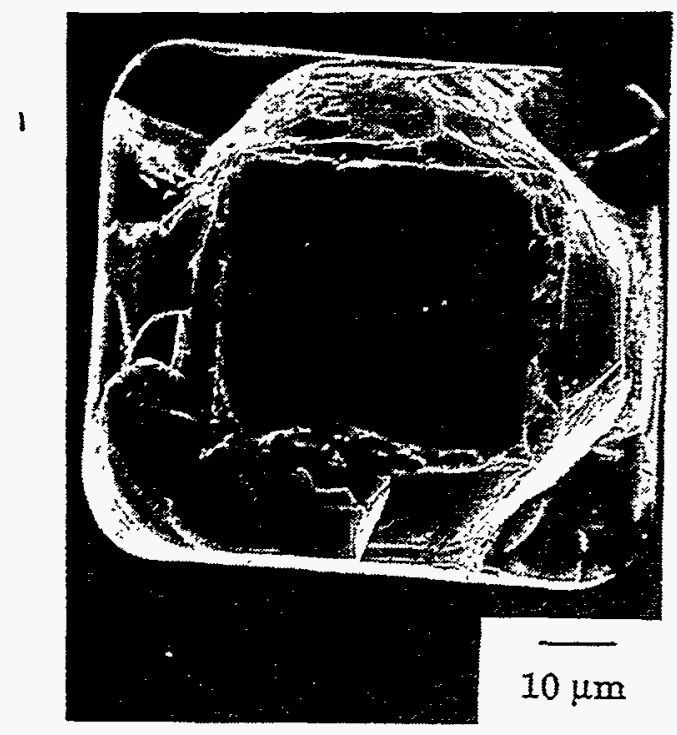

(a)

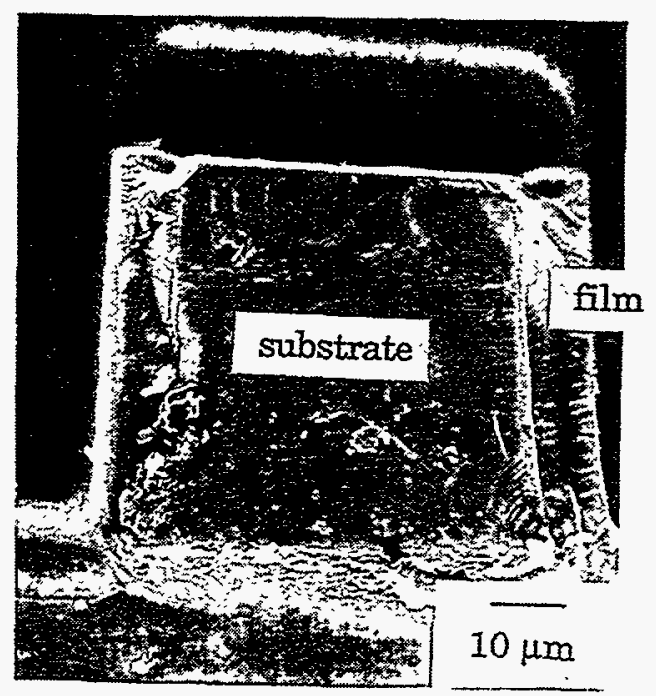

(b)

Fig. 4 Scanning electron micrographs of mesa-patterned samples where some films peeled off. (a) Top view of the delaminated mesa, (b) Fractured cross section of the partially peeled sample. Films on other mesas are seen in the background. 


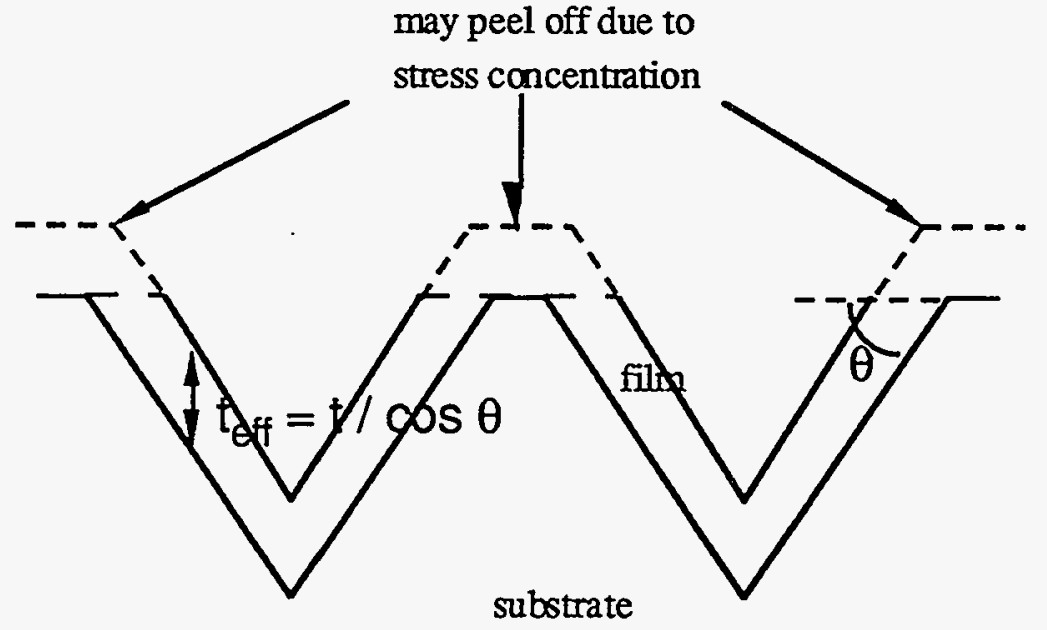

Fig. 5. Schematic diagram of the cross section of the inversed pyramid pattern

was decreased to $40 \mu \mathrm{m}$. However, such small patterns are not practical because the trench area is larger than the active area. Also, the stress analysis can no more be approximated as 2dimensional and is more complicated. A-Si:H films of up to $20 \mu \mathrm{m}$ in thickness were deposited successfully, but thicker films partially peeled off. The SEM photographs of a-Si:H films grown on such structures are shown in Fig. 4. Cracks developed around the edges of the mesas due to the high stress concentration on sharp corners, and the films on the top of the mesas buckled. However, the

films inside the trench were more stable than those at the top. On plain substrates, films of up to $20 \mu \mathrm{m}$ in thickness remained stable with the residual stress of $350 \mathrm{MPa}$. It is observed in fig. 4 that films of about half the thickness of the top ones are deposited quite uniformly on the sidewalls and at the bottom of the trenches. Although the top part peeled off, the films inside the grooves remained attached firmly. In summary, the selective area deposition helps reduce the substrate bending, but cannot prevent the peel-off. Also, it is suggested that the use of the films inside the trenches might be safer than of the top portion.

\section{Inversed Pyramid Patterns}

As discussed in the previous section, the films inside the trenches appear to be mechanically more stable. Therefore, it would be beneficial to confine films in the trenches or wells rather than to have them on the pedestal. However, it is very difficult to deposit films only at the bottom of the trenches or on the vertical wall and to make reliable electrical contacts on them. An alternative way is to grow films on the slopes of V-shaped holes as shown in Fig. 5. In the pyramid structure, the horizontal component of the stress, which is related to the bending moment, is decreased by $\cos \theta$, where $\theta$ is the angle between the horizontal surface and the side, and thus substrate bending will be reduced. Delamination of the films will be minimized as well since the films are confined inside the inversed pyramids. It is like a piece of filter paper sitting inside a funnel. The paper tends to expand outward and is tightly bound to the funnel.

The pyramid structure may also enhance the signal collection in two ways. First, the effective thickness of the film through which the radiation particles travel is increased by $1 / \cos \theta$, hence full $50 \mu \mathrm{m}$ deposition will be unnecessary. Second, the direction of the charge carrier sweep-out is inclined to the incident particle by an angle $\theta$, and thus the recombination of the electrons and holes along the particle track is reduced. This effect was demonstrated with plain geometry a-Si:H diodes. ${ }^{19)}$ When the detector was tilted at $60^{\circ}$ to the incident particle, the collected signal increased by a factor of 2 .

The inversed pyramid structure can be realized by anisotropic etching of the single crystal silicon wafers. First, $1 \mu \mathrm{m}$-thick oxide was grown on a $<100>$ orientation wafer in $1100^{\circ} \mathrm{C}$ pyrogenic steam furnace as a masking layer. $180 \mu \mathrm{m}$ x $180 \mu \mathrm{m}$ squares with $20 \mu \mathrm{m}$ spacing were patterned by photolithography, aligning the major flat of the wafer with a side of the square. The backside of the wafer was coated with photoresist to protect the oxide layer, and the patterned-side 
oxide layer was etched with 5:1 buffered HF. The wafer was then etched in EDP solution (500 ml of ethylenediamine, $160 \mathrm{~g}$ of pyrocatecol, $3 \mathrm{~g}$ of pyrazine dissolved in $160 \mathrm{ml}$ of $\mathrm{H}_{2} \mathrm{O}$ ) until all (111) surfaces were exposed. Etch rate of $(100)$ plane is around $1 \mu \mathrm{m} / \mathrm{min}$. at $105^{\circ} \mathrm{C}$ and the etch rate ratio of $\langle 100\rangle$ : $\langle 110\rangle:\langle 111\rangle$ orientation is 50:30:3. All the remaining oxides were stripped and the patterned surface was coated with $\mathrm{Cr}$. A-Si:H films of $15 \mu \mathrm{m}$ and $32 \mu \mathrm{m}$ in thickness were deposited and the stress and the fracture cross section were observed.

Fig. 6 shows the SEM pictures of inversed pyramid pattern engraved in a silicon wafer by anisotropic and a-Si:H film deposited inside the pyramids. As seen in the he films on the dead area between the pyramids peeled off because of the large stress concentration as discussed in the previous section. However, this effect is beneficial because it further releases the stress and isolates pixels from each other. The measured apparent stress value was around $100 \mathrm{MPa}$ which is smaller than that of the normal substrate by a factor of $3 \sim 4$.

A computer simulation was performed using the identical geometry and material parameters as those of an actual sample. Fig. 7 shows a cross sectional view of the stress distribution with a color scale. Fig. 7(a) represents a sample with the a-Si:H film deposited evenly on the pyramid patterned substrate, and fig. 7(b) shows a sample with the top portion of the film peeled off due to the stress concentration the sharp peaks. The left side of the substrate was assumed to be fixed to the wall and all the other parts were allowed to deform freely. As illustrated by colors in the figure, most of the stress is taken up by the pyramid part and only a small stress appears in the back of the substrate. If carefully observed, slightly more deformation is noticed in the substrate with all films remained intact (fig. 7(a)) than in the substrate with the top portion peeled off (fig. 7(b)).

According to these results, the inversed pyramid structure is preferred to the square island pattern for films under high compression. However, there are also some drawbacks in this structure. First, a large stress concentration occurs at the sharp point in the valley of the pyramid as well as near the top and weakens the substrate. This large tensile stress concentrated near the bottom of the pattern can also be visualized by a red color in fig. 7(a). This effect can be moderated to some extent by decreasing the curvature of the point. If a short isotropic etching follows the anisotropic etching, it will decrease the curvature appreciably without degrading the slope much. It will also reduce the intense electric field concentration around the sharp corner which might cause electrical breakdown. Second, cross-section of the film is exposed at the top of the pyramid due to the peel-off, and there might be an arc across the surface when a high bias voltage is applied. Therefore, the exposed cross-section needs to be covered by a proper insulator.

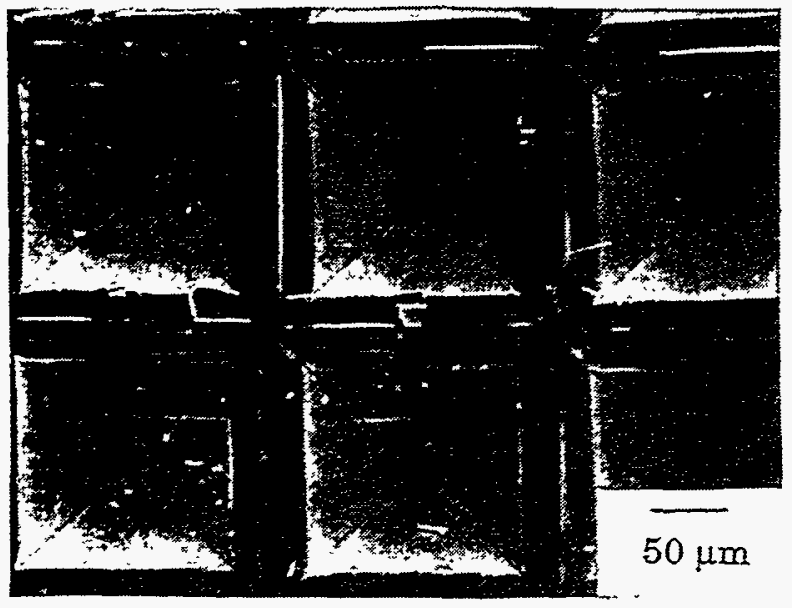

(a)

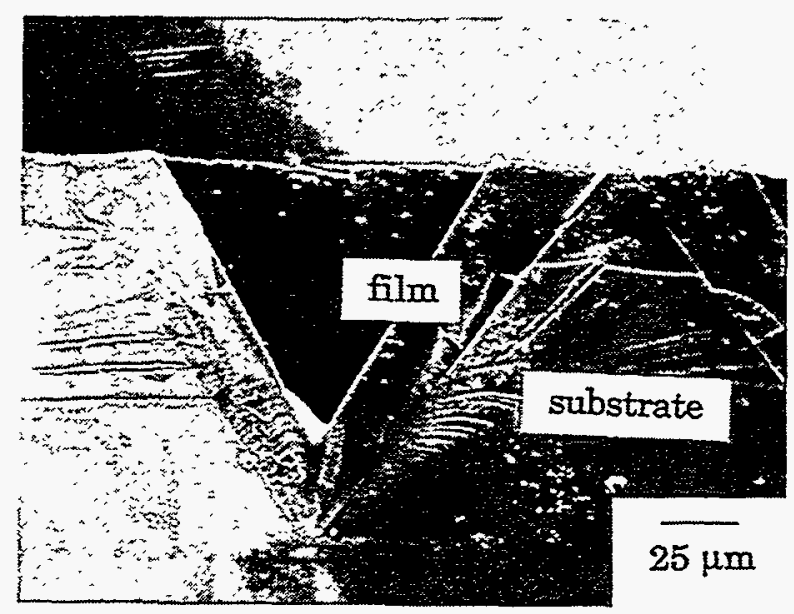

(b)

Fig. 6. Scanning electron micrographs of inversed pyramid patterns: (a) Top view of $20 \mu \mathrm{m}$-thick a-Si:H films deposited inside the V-shaped holes, (b) Fractured cross section of $32 \mu \mathrm{m}$-thick a-Si:H film deposited on the pyramid patterned substrate. 

One of the best candidates is polyimide because it can be easily spin coated and patterned by UV light.

\section{SUMMARY}

Deposition on the patterned substrates was attempted to minimize delamination and substrate bending without changing the deposition parameters. A-Si:H films deposited on square islands showed that the apparent stress lowered by $40 \%$ for the island width of $40 \mu \mathrm{m}$, compared to those on the flat surface. However, when the films are thicker than $20 \mu \mathrm{m}$, some of them peeled off at the top of the mesas. When the inversed pyramid pattern of $200 \mu \mathrm{m}$ in width was used instead of square mesas, $32 \mu \mathrm{m}$-thick films could be deposited without delamination. The top portion of the film between the pixels peeled off due to the stress concentration, releasing the stress further and isolating pixels from each other. The apparent stress was reduced to by a factor of 3 4. Computer simulation showed that the stress was imposed mostly on the patterned region, and the rest of the substrate experienced less stress. Inversed pyramid structure seemed to be better than the square mesa structure in the mechanical stability point of view, although it still needs to be improved.

(a)

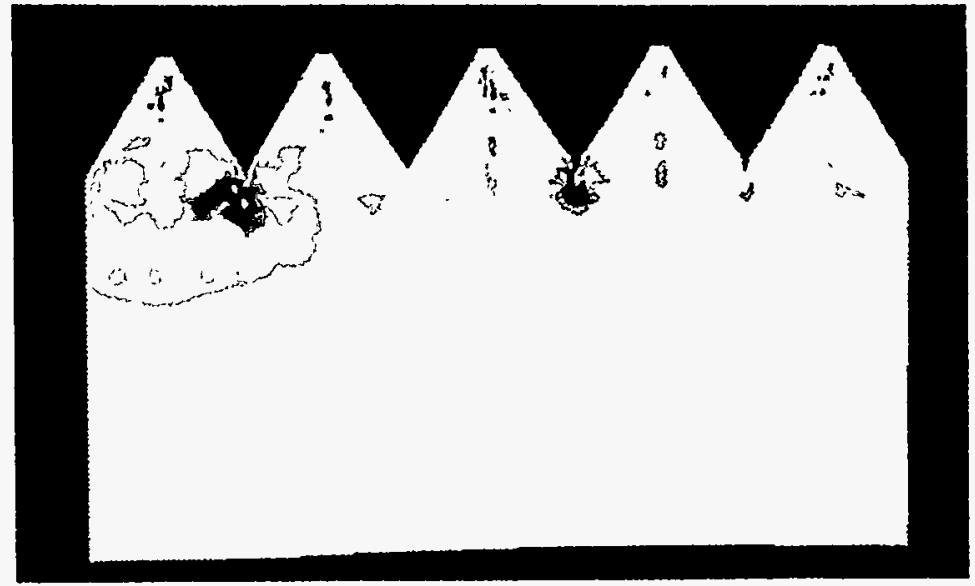

(b)
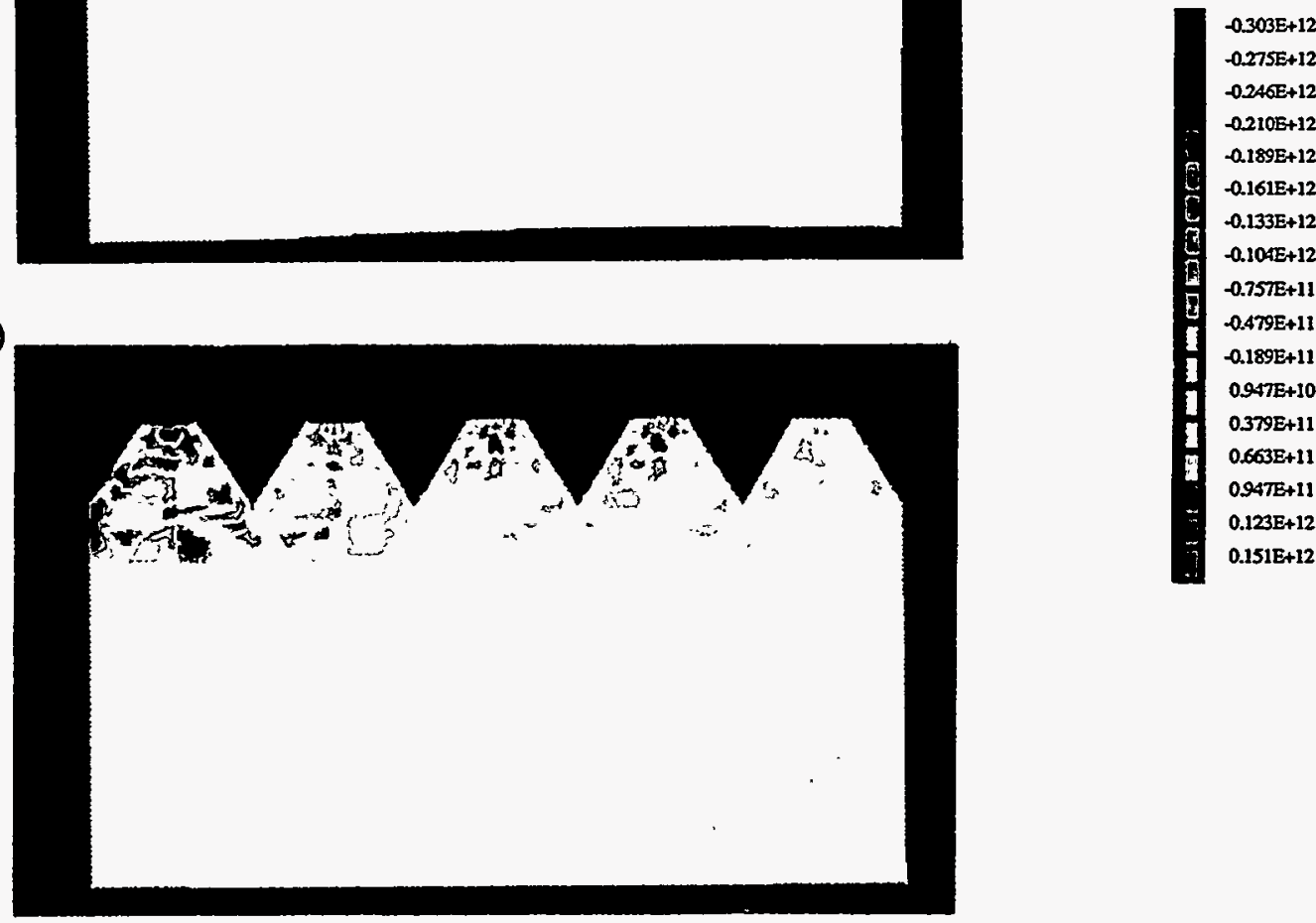

Fig. 7. Simulation results of the stress distribution in the pyramid-patterned substrate. The yellow-green color represents zero stress and color change toward red indicates increasing tensile stress. The unit of the stress is dynes $/ \mathrm{cm} 2$. (a) When all films remained attached on the substrate, (b) When the top portion peeled off. 


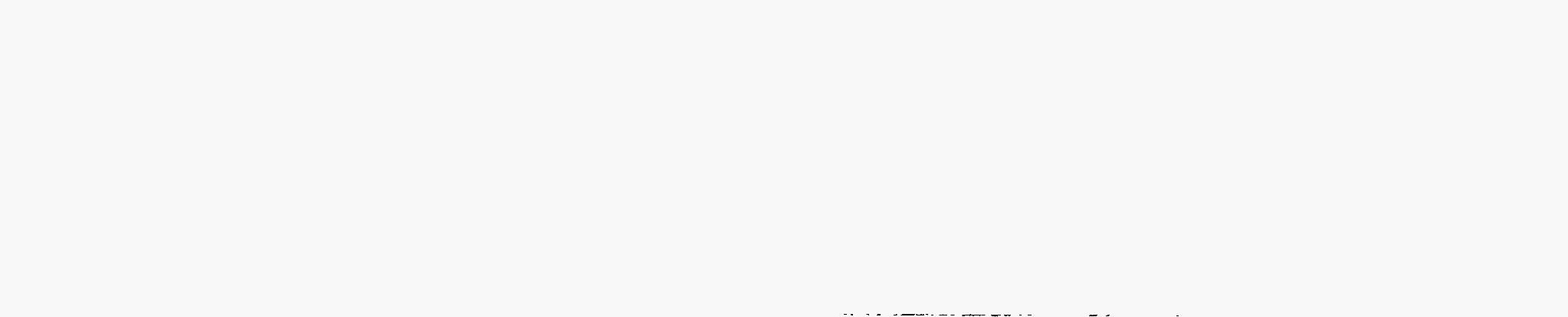


larger area application and for lower cost. An alternative way to produce a-Si:H films of good qulaity at reduced stress is to grow at low temperature $\left(<160^{\circ} \mathrm{C}\right)$ and anneal. Proper annealing turns out to restore the increased dangling bond density without affecting the stress, but it requires more investigation.

\section{ACKNOWLEDGMENT}

This work was supported by the Director, Office of Energy Research, Office" of High Energy Nuclear Physics, High Energy Physics Division of U.S.Department of Energy under Contract No. DE-AC03-76SF00098.

\section{REFERENCES}

1) V.Perez-Mendez, G.Cho, J.Drewery, T.Jing, S.N.Kaplan, S.Qureshi, D. Wildermuth, C.Goodman, I.Fujieda and R.A.Street, Nucl. Phys. B, 32, 287 (1993)

2) B.Equer and A.Kara, Nucl. Instr. Meth. A271, 574 (1988)

3) J.Dubeau, T.Pochet, A.Karar, L.A.Hamel, B.Equer, J.P.Martin, S.C.Gujrathi and A.Yelon in Amorphous Silicon Technology, edited by A.Madan, M.J.Thompson, P.C.Taylor, P.G.LeComber, Y.Hamakawa (Mater. Res. Soc. Proc. 118, Pittsburgh, PA, 1988) pp.439444.

4) J.S.Drewery, G.Cho, I.Fujieda, T.Jing, S.N.Kaplan, V.Perez-Mendez and D.Wildermuth, Nucl. Instr. Meth. A310, 165 (1991)

5) H.Kakinuma, S.Nishikawa, T.Watanabe and K.Nihei, J. Appl. Phys. 5923110 (1986)

6) J.P.Harbison, A.J.Williams and D.V.Lang, J. Appl. Phys. 554946 (1984)

7) K.S.Stevens and N.M.Johnson, J. Appl. Phys. $71 \underline{6} 2628$ (1992)

8) N.M.Johnson, F.A.Ponce, R.A.Street and R.J.Nemanich, Phys. Rev. B 35816166 (1987)

9) N.M.Johnson, C.Doland, F.Ponce, J.Walker and G.Anderson, Physica B, 170, 3 (1991)

10) R.A.Street, Hydrogenated Amorphous Silicon, (Cambridge Univ. Press, Cambridge, 1991),

11) G.Stoney, Proc. Roy. Soc. London Ser. A, 82, 172 (1909)

12) S.Qureshi, V.Perez-Mendez, S.N.Kaplan, I.Fujieda, G.Cho and R.A.Street, IEEE Trans. Nucl. Sci. 361194 (1989)

13) W.E.Spear and M.Heinze, Phil. Mag. B, 545343 (1986)

14) Y.Hishikawa, J. Appl. Phys. $62 \underline{8} 3150$ (1987)

15) W.S.Hong, A.Mireshghi, J.S.Drewery, T.Jing, Y.Kitsuno, H.K.Lee, S.N.Kaplan and V.Perez-Mendez, Presented at the IEEE Nucl. Sci. Symp., Norfolk, VA, Oct. 31-Nov. 5, 1994, and to be published in the IEEE Trans. Nucl. Sci.

16) M.Yamaguchi, M.Tachikawa, M.Sugo, S.Kondo and Y.Itoh, Appl. Phys. Lett. 56127 (1990)

17) B.Yacobi, S.Zemon, P.Norris and C.Jagannath P.Sheldon, Appl. Phys. Lett. $51 \underline{26} 2236$ (1987)

18) E.Suhir, J. Appl. Mech. 53657 (1986)

19) S.Qureshi, Hydrogenated Amorphous Silicon Radiation Detectors: Material Parameters: Radiation Hardness: Charge Collection. Ph.D. Thesis, Univ. of Calif., Berkeley, (1991) 